\title{
Lessons Learned From Industrial Validation of COSYSMO
}

17th INCOSE Symposium

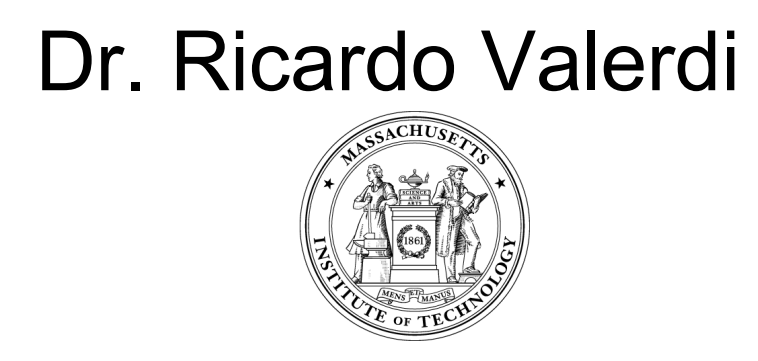

Garrv Roedler
Marilee Wheaton

(A) THE AEROSPACE

Dr. John Rieff
LOCKHEED MA RTIN
iaytheon 


\section{Outline}

- Introduction \& motivation

- Summary of lessons from model development (11)

- Industrial validation of COSYSMO (15)

- Application challenges

- The future 


\section{Introduction \& Motivation}

- Constructive Systems Engineering Cost Model (COSYSMO)

- COCOMO II family

- Development began in 2001

- Extensive practitioner support

- ISPA, INCOSE, CSSE Corporate Affiliates, LAI Consortium

- Historical project data \& industry calibration enables

- understanding the model's robustness

- establishment of initial relationships between parameters and outcomes

- validation of drivers

- Challenge is that SE measurement is not standardized

- Model development process yielded 11 lessons learned

Valerdi, R., Rieff, J., Roedler, G., Wheaton, M., Lessons Learned from Collecting Systems Engineering Data, Conference on Systems Engineering Research, April 2004. 


\section{Lessons Learned from Development}

Lesson \#1: Scope of the model

Lesson \#2: Types of projects needed for data collection effort

Lesson \#3: Size drivers

Lesson \#4: Effort Multiplier

Lesson \#5: Systems Engineering hours across life cycle stages

Lesson \#6: Data collection form

Lesson \#7: Definition

Lesson \#8: Significance vs. data availability

Lesson \#9: Influence of data on the drivers and statistical significance

Lesson \#10: Data safeguarding procedure

Lesson \#11: Buy-in from constituents 


\section{Lessons Learned from Validation}

Lesson \#1: Skills Needed to use COSYSMO

Provide a list of assumptions/prerequisites for model use as well as the appropriate training/resources for COSYSMO understanding

\section{Lesson \#2: Model Usability}

Understanding usability will lead to more reliable inputs to the model especially at the early phases of the life cycle where there is little project information available

\section{Lesson \#3: Model Adoption}

Providing organizations with a sequential process driven by implementation experience will facilitate the adoption of COSYSMO

Lesson \#4: Accounting for Reuse

Providing a way to account for reuse in systems engineering is essential for improving the accuracy of the model 


\section{Lessons Learned from Validation}

\section{Lesson \#5: Risk in Cost Estimates}

Modeling the probability of the estimate provided by COSYSMO will help assess the risk associated with that estimate as part of the overall risk management strategy for the project

\section{Lesson \#6: Counting Rules}

Detailed counting rules can ensure that size drivers, specifically requirements, are counted consistently across the diverse set of systems engineering projects, hence improving the model's application across organizations

\section{Lesson \#7: Rating Complexity}

Guidance on rating complexity via easy, nominal, difficult is necessary to ensure consistent use across organizations

\section{Lesson \#8: Rating Drivers With Multiple Viewpoints}

Clarification on how rating levels are averaged between multiple viewpoints is needed to reconcile possible conflicts in the driver interpretations 


\section{Lessons Learned from Validation}

Lesson \#9: Driver Rating Scales and Polarization

Matching the ratings scales and polarization of drivers made their impact on systems engineering effort easier to understand

Lesson \#10: Overlap of Requirements, Operational Scenarios, Algorithms, Interfaces

Detailed examples need to be provided to prevent double dipping across multiple size drivers

\section{Lesson \#11: Effect of Schedule on Effort}

Systems engineering schedule is driven by project-level milestones and is therefore unlike the cube root law in software

\section{Lesson \#12: Life Cycle Coverage}

Focus the scope of COSYSMO only on life cycle phases that can be calibrated with historical data 


\section{Lessons Learned from Validation}

Lesson \#13: Systems Engineering Effort Profile

The capability to model systems engineering effort distribution by phase is necessary since many projects estimate portions of the life cycle rather than the entire lifecycle

\section{Lesson \#14: Local Calibration}

Provide ways for individual organizations to self-calibrate COSYSMO

\section{Lesson \#15: Prototypes}

Defining prototypical system types will help communicate the application of the model 


\section{Application Challenges of COSYSMO}

- Requirements Change throughout system life cycle

- Difficult to quantify impact of requirements volatility on SE effort

- Estimation needs are more complex than what the data provides

- Standard WBS vs. detailed systems engineering tasks

- Local calibrations are time consuming

- Data from multiple homogenous programs is needed to generate a useful calibration 


\section{The Future of COSYSMO}

- Continued data collection with LMCO, BAE, Raytheon, SAIC, General Dynamics, Northrop Grumman, L-3 Communications

- Boeing is next

- Commercial versions available from: Galorath, Inc., PRICE Systems, SoftStar Systems

- COSYSMO book (Wiley 2007)

- "Systems Engineering Cost Estimation with COSYSMO"

- COSYSMO documentation available at: http://www.valerdi.com/cosysmo 


\section{References}

Miller, C., Measuring Usability in COSYSMO, PhD Dissertation Proposal, George Washington University, Spring 2006.

Roedler, G., "Adapting COSYSMO to Accommodate Reuse." LMCO Measurement Workshop, Valley Forge, PA, September 2006.

Valerdi, R., Rieff, J., Roedler, G., Wheaton, M., Lessons Learned from Collecting Systems Engineering Data, Conference on Systems Engineering Research, April 2004.

Valerdi, R., Ernstoff, M., Mohlman, P., Reifer, D., Stump, E., Systems Engineering Sizing in the Age of Acquisition Reform, 18th Annual Forum on COCOMO and Software Cost Models, October 2003, Los Angeles, CA.

Valerdi, R., Boehm, B., Reifer, D., COSYSMO: A Constructive Systems Engineering Cost Model Coming Age, Proceedings, 13th Annual International INCOSE Symposium, July 2003, Crystal City, VA 